\title{
Dor torácica no infarto agudo do miocárdio entre pacientes diabéticos e não diabéticos
}

\author{
Chest pain in acute myocardial infarction among diabetic and non-diabetic patients
}

Dolor torácico en el infarto agudo de miocardio entre pacientes diabéticos y no diabéticos

\section{Crislaine Padilha Paim', Karina de Oliveira Azzolin", Maria Antonieta Pereira de Moraes" \\ ' Fundação Universitária de Cardiologia, Instituto de Cardiologia do Rio Grande do Sul. Universidade Federal do Rio Grande do Sul; Hospital de Clínicas de Porto Alegre. Porto Alegre-RS, Brasil. \\ "Fundação Universitária de Cardiologia, Instituto de Cardiologia do Rio Grande do Sul, Programa de Pós Graduação. Porto Alegre-RS, Brasil.}

Submissão 13-07-2010 Aprovação: 02-03-2012

\section{RESUMO}

Considerando-se que pacientes diabéticos acometidos de Infarto Agudo do Miocárdio (IAM) podem, ou não, apresentar dor torácica, objetivou-se neste estudo comparar a presença e intensidade de dor torácica no IAM entre pacientes diabéticos e não diabéticos. Realizou-se um estudo transversal, que incluiu pacientes com IAM, idade $\geq 18$ anos, ambos os sexos. Utilizou-se uma escala numérica verbal para avaliar a presença e a intensidade da dor. Foram incluídos 88 pacientes no estudo, dentre os quais $77(87,5 \%)$ não diabéticos e $11(12,5 \%)$ diabéticos. A dor esteve presente em $11(100 \%)$ dos diabéticos e $76(98,7 \%)$ dos não diabéticos. A intensidade da dor nos diabéticos foi de 8,91 versus 8,23 nos não diabéticos. O estudo mostrou semelhança na presença e intensidade da dor torácica entre pacientes diabéticos e não diabéticos acometidos de IAM.

Descritores: Diabetes mellitus; Infarto do miocárdio; Escala analógica da dor.

\section{ABSTRACT}

Considering that diabetic patients suffering from Acute Myocardial Infarction (AMI) may or may not have chest pain, this study aimed to compare the presence and intensity of chest pain in AMI between diabetic and non-diabetic patients. We conducted a cross-sectional study that included patients with AMI, aged $\geq 18$ years, both sexes. We used a verbal numeric scale for assessing the presence and intensity of pain. The study included 88 patients, of whom $77(87.5 \%)$ non-diabetic patients and $11(12.5 \%)$ diabetics. The pain was present in $11(100 \%)$ of diabetics and in $76(98.7 \%)$ of non-diabetics. The intensity of pain in diabetics was 8.91 versus 8.23 in non-diabetic patients. The study showed similarity in the presence and intensity of chest pain between diabetic and non diabetic patients suffering from AMI.

Key words: Diabetes mellitus; Myocardial infarction; Analogical score of pain.

\section{RESUMEN}

Teniendo en cuenta que los pacientes diabéticos acometidos de Infarto Agudo del Miocardio (IAM) pueden o no presentar dolor torácico. Este estudio objetivó comparar la presencia e intensidad de dolor torácico en el IAM entre pacientes diabéticos y no-diabéticos. Realizó-se un estudio transversal, que incluyó a pacientes con IAM, ambos sexos, edad $\geq 18$ años. La presencia y la intensidad del dolor fueron evaluadas a través de la escala numérica verbal. Se incluyeron 88 pacientes, de los cuales 77 $(87,5 \%)$ no-diabéticos y $11(12,5 \%)$ diabéticos. El dolor estuvo presente en $11(100 \%)$ de los diabéticos y en $76(98,7 \%)$ de los no-diabéticos. La intensidad del dolor en los diabéticos fue de 8,91 versus 8,23 en no-diabéticos. El estudio mostró igualdades en la presencia e intensidad del dolor torácico entre pacientes diabéticos y no-diabéticos acometidos de IAM.

Palabras clave: Diabetes mellitus; Infarto de miocardio; Escala analógica del dolor. 


\section{INTRODUÇÃO}

O diabetes mellitus (DM) representa a quinta causa de morte no mundo ${ }^{(1)}$ e dentre as suas complicações, sabe-se que as doenças cardiovasculares ocorrem com maior frequência, sendo também responsáveis por esta elevada mortalidade. Dentre as doenças cardiovasculares, a doença arterial coronariana (DAC) é uma das mais prevalentes, a qual compreende o Infarto Agudo do Miocárdio (IAM) e a Angina Instável.

A ocorrência em larga escala da DAC em pacientes com diabetes tem sido reconhecida há muito tempo. Estudo epidemiológico mostrou que, em pacientes diabéticos, a incidência de DAC é duas vezes maior nos homens e três vezes maior nas mulheres, quando comparados com a população em ge$\mathrm{ral}^{(2)}$. As comorbidades associadas ao DM elevam o risco cardiovascular e exigem esforços redobrados para a prevenção dos eventos maiores ${ }^{(3)}$. É de amplo conhecimento a origem multifatorial da DAC, fato comprovado pelo estudo europeu INTERHEART, conduzido em cinco continentes, que avaliou os fatores de risco para o IAM, demonstrando que dislipidemia, tabagismo, diabete mellitus, hipertensão arterial, obesidade abdominal, fatores psicossociais, baixo consumo diário de frutas e vegetais, sedentarismo e consumo de álcool explicam $90 \%$ do risco para o IAM, em ambos os sexos, em todas as faixas etárias e em todas as partes do mundo ${ }^{(4)}$.

O maior estudo epidemiológico brasileiro que avaliou os fatores de risco associados ao IAM no Brasil foi o AFIRMAR, publicado em 2003. A principal diferença encontrada em relação ao INTERHEART foi a ordem de importância de alguns fatores de risco; contudo, o DM permaneceu sendo o terceiro fator de risco mais importante nos dois estudos ${ }^{(5)}$.

A manifestação clínica mais comum da DAC aguda é a dor precordial, grave ou transitória( ${ }^{(6)}$ e, na maioria das vezes, em evolução o IAM. Porém, no indivíduo diabético podem ocorrer sinais e sintomas atípicos, provavelmente devido à neuropatia sensitiva e autonômica; assim, podem prevalecer sintomas como dispnéia, sudorese, náuseas e vômitos, levando a uma postergação da procura de um serviço de emergência(7).

Em estudo que teve como objetivo comparar a dor isquêmica do IAM, entre pacientes diabéticos e não diabéticos, foram analisados 80 pacientes, sendo que 23 tinham diabetes e 57 não tinham diabetes. Os resultados demonstraram que somente três pacientes diabéticos tiveram ausência de dor, ou seja, $87 \%$ dos pacientes diabéticos e $100 \%$ dos não diabéticos apresentaram dor torácica típica na vigência do IAM ${ }^{(8)}$.

Evidências indiretas de estudos clínicos de pesquisas epidemiológicas vêem contrapor a idéia de ausência ou diminuição da dor nestes sujeitos, sugerindo que o aumento na prevalência de doença coronariana assintomática nos diabéticos não difere significativamente do aumento das formas sintomáticas do evento. Sendo assim, a neuropatia não poderia ser relacionada com a maior parte da falta de dor isquêmica, uma vez que os mecanismos que geram esta dor são complexos e controversos mesmo sem a presença do diabetes ${ }^{(9,10)}$.

Partindo dessas considerações, este estudo objetivou comparar a presença e intensidade de dor torácica no infarto agudo do miocárdio entre pacientes diabéticos e não diabéticos, com o intuito de melhorar a avaliação e assistência da equipe de saúde frente a estes pacientes.

\section{MÉTODOS}

Trata-se de um estudo com delineamento transversal prospectivo, realizado na Sala de Emergência (SE) de um hospital cardiológico do Sul do País. Foram incluídos pacientes com diagnóstico de IAM com ou sem supra-desnivelamento de ST, em até 24 horas da admissão na SE, presença ou não de diabete mellitus, de ambos os sexos, com idade $\geq 18$ anos. Foram excluídos pacientes com sequelas cognitivas, visuais e em uso de qualquer tipo de analgesia contínua.

Com base no estudo realizado por Gondim e cols. ${ }^{(8)}$, e considerando um erro $\alpha$ de $5 \%$ e poder de $80 \%$, para que fosse possível detectar uma diferença de 1,5 pontos na nota de dor entre diabéticos e não diabéticos (desvio padrão 2,8 e 2,0 respectivamente), estimamos o tamanho da amostra em 88 pacientes.

Os dados foram coletados através de entrevistas com os pacientes e análise dos registros da equipe de saúde nos prontuários. Um instrumento foi elaborado para este estudo, contendo variáveis sociais, clínicas e demográficas, histórico de doença pregressa, história da doença atual, características da dor (intensidade, localização, qualidade, irradiação) e sintomas associados.

Os pacientes com diagnóstico de IAM foram divididos em dois grupos: com e sem diabetes. Os pacientes diabéticos deveriam ter diagnóstico prévio da doença e fazer uso de drogas hipoglicemiantes orais ou insulina. Os critérios diagnósticos para IAM e diabetes foram os utilizados na instituição e que seguem os guidelines preconizados, respectivamente, pela American Heart Association (AHA) ${ }^{(11)}$ e pela American Diabetes Association (ADA) ${ }^{(12)}$.

A presença e a intensidade da dor foram avaliadas pela aplicação de escala numérica verbal, na chegada do paciente à sala de emergência. Esta escala é validada e consiste em classificar a dor em valores numéricos de zero a 10, tendo como parâmetros que zero é igual à ausência de dor e 10 a maior dor sentida pelo paciente em toda a sua vida. A dor é classificada em níveis de intensidade fraca (1-3), moderada (4-6), forte (7-9) e insuportável (10) ${ }^{(13)}$. Para evitar o viés de aferição, duas enfermeiras treinadas entrevistaram os pacientes e aplicaram a escala da dor.

O projeto foi submetido e aprovado pelo Comitê de Ética em Pesquisa da Instituição (Protocolo n 3.987/07) e somente participaram do estudo os pacientes que assinaram o termo de consentimento livre e esclarecido. Os dados foram analisados com auxílio do programa estatístico Statistical Package for Social Sciences (SPSS) 14.0. As variáveis categóricas foram expressas com frequências absolutas e relativas, e as variáveis contínuas descritas com média e desvio padrão ou mediana e intervalo interquartílico (percentil 25 e 75). Para a comparação das variáveis categóricas entre os grupos foi utilizado o teste exato de Fisher e para as variáveis contínuas, os testes t de Student ou teste de Mann-Whitney. O valor de $\mathrm{p}<0,05$ foi considerado estatisticamente significante. 


\section{RESULTADOS}

Foram incluídos neste estudo 88 pacientes, dos quais 77 $(87,5 \%)$ eram não diabéticos e 11 (12,5\%) diabéticos, com média de idade de $58,2 \pm 10,6$ anos e predomínio do sexo masculino 57 (64,7\%). Dados estão expressos na Tabela 1.

\section{Presença e Característica da dor}

Os resultados demonstraram que a dor esteve presente em $11(100 \%)$ diabéticos e em $76(98,7 \%)$ dos pacientes não diabéticos, sendo que os diabéticos apresentaram percentual maior de dor de forte intensidade $(63,7 \%$ vs $44,2 \%)$ e menor de dor insuportável (36,4\% vs $44,2 \%$ ) quando comparados aos pacientes não diabéticos. Ao avaliar a intensidade média da dor entre os dois grupos, observou-se que, nos diabéticos, foi de 8,91 e, nos não diabéticos, de 8,23 , não apresentando diferença estatisticamente significativa $(p<0,278)$. Quanto às características da dor referida pelos pacientes, predominaram queixas de dor retroesternal $(57-64,7 \%)$, em queimação $(46-52,2 \%)$ e irradiação para o membro superior esquerdo $(54-61,3 \%)$. As manifestações clínicas mais citadas foram sudorese $(57-64,7 \%)$, seguido de náuseas ( 38 - 43,1\%). Não houve associação estatisticamente significante entre escala da dor e tempo de diabetes ( $p$ $=0,142)$. Estes dados estão expressos na Tabela 2 .

\section{DISCUSSÃO}

O presente estudo comparou a presença e a intensidade da dor torácica no IAM entre pacientes diabéticos e não diabéticos, por meio da aplicação da escala numérica verbal. $\mathrm{Na}$ amostra, $100 \%$ dos diabéticos sentiram dor, comparado $98,7 \%$ dos não diabéticos, demonstrando assim, não haver diferenciação da dor entre ambos os grupos.

Estudos consagrados, como a coorte de Framingham ${ }^{(2)}$, demonstram que há um aumento do risco de doenças cardiovasculares em pacientes que apresentam glicose alterada em jejum e após o teste de tolerância à glicose. Sabe-se que a glicose não controlada, com o passar do tempo, pode levar a complicação do diabetes como doenças micro e macrovasculares, retinopatia, nefropatia e neuropatia autonômica. Essas complicações provocariam alterações na transmissão ou geração de impulsos nervosos entre o sistema nervoso central $(\mathrm{SNC})$ e a periferia do paciente, tendo como resultado a redução ou até mesmo a ausência de dor, conhecida como isquemia silenciosa no $\mathrm{IAM}^{(2,8)}$.

A dor nos últimos anos tem sido valorizada e muitos autores a consideram como o quinto sinal vital. Escalas de mensuração foram criadas e validadas no intuito de quantificar e qualificar a dor, sendo largamente utilizadas, apesar de a dor

Tabela 1 - Características sócio-demográficas e clínicas da amostra.

\begin{tabular}{|c|c|c|c|}
\hline & $\begin{array}{c}\text { Não diabéticos } \\
\mathrm{n}=77\end{array}$ & $\begin{array}{c}\text { Diabéticos } \\
\mathrm{n}=11\end{array}$ & $P$ \\
\hline Idade (anos)* & $57,66 \pm 10,74$ & $62,27 \pm 9,35$ & $0,181^{\bullet}$ \\
\hline Sexo masculino $^{+}$ & $49(63,6 \%)$ & $8(72,7 \%)$ & $0,740^{\alpha}$ \\
\hline Raça branca & $63(81,8 \%)$ & $9(81,8 \%)$ & $1,000^{\alpha}$ \\
\hline Índice massa corporal & $26,1 \pm 3,9$ & $29,9 \pm 5,7$ & $0,006^{\bullet}$ \\
\hline Pressa arterial sistólica (mm/Hg) & $138,0 \pm 30,8$ & $149,0 \pm 38,5$ & $0,287^{\bullet}$ \\
\hline Pressão arterial diastólica $(\mathrm{mm} / \mathrm{Hg})$ & $83,4 \pm 18,0$ & $88,1 \pm 20,4$ & $0,429^{\circ}$ \\
\hline Frequência cardíaca (bpm) & $76,7 \pm 14,9$ & $80,1 \pm 21,4$ & $0,504^{\bullet}$ \\
\hline \multicolumn{4}{|l|}{ Comorbidades } \\
\hline Infarto agudo miocárdio & $12(15,6 \%)$ & $2(18,2 \%)$ & $1,000^{\alpha}$ \\
\hline Insuficiência cardíaca & $1(1,3 \%)$ & $0(0 \%)$ & $1,000^{\alpha}$ \\
\hline Tabagismo & $51(66,2 \%)$ & $8(72,7 \%)$ & $1,000^{\alpha}$ \\
\hline Hipertensão arterial sistêmica & $45(58,4 \%)$ & $9(81,8 \%)$ & $0,191^{\alpha}$ \\
\hline Dislipidemia & $30(39,0 \%)$ & $4(36,4 \%)$ & $1,000^{\alpha}$ \\
\hline História familiar positiva & $49(63,6 \%)$ & $9(81,8 \%)$ & $0,319^{\alpha}$ \\
\hline Doença cerebrovascular & $5(6,5 \%)$ & $1(9,1 \%)$ & $0,563^{\alpha}$ \\
\hline Doença vascular periférica & $4(5,2 \%)$ & $1(9,1 \%)$ & $0,496^{\alpha}$ \\
\hline Revascularização percutânea & $8(10,4 \%)$ & $1(9,1 \%)$ & $1,000^{\alpha}$ \\
\hline Revascularização cirúrgica & $3(3,9 \%)$ & $0(0 \%)$ & $1,000^{\alpha}$ \\
\hline Tempo dor até SE (minutos) & $240(135-405)$ & $300(180-1020)$ & $0,293^{\wedge}$ \\
\hline \multicolumn{4}{|c|}{$\begin{array}{l}\text { Legenda: } \\
\text { † Variáveis categóricas expressas como n (\%). } \\
\text { * Variáveis contínuas como média } \pm \text { desvio padrão. } \\
\text { - P nível descritivo do teste } t \text { de Student. } \\
\text { a P nível descritivo do teste exato de Fisher. }\end{array}$} \\
\hline
\end{tabular}


Tabela 2 - Características da dor.

\begin{tabular}{|c|c|c|c|}
\hline & $\begin{array}{c}\text { Não diabéticos } \\
n=77\end{array}$ & $\begin{array}{c}\text { Diabéticos } \\
n=11\end{array}$ & $P^{\alpha}$ \\
\hline \multicolumn{4}{|l|}{ Localização: } \\
\hline Retroesternal & $49(63,6 \%)$ & $8(72,7 \%)$ & 0,740 \\
\hline Precordial & $34(44,2 \%)$ & $5(45,5 \%)$ & 1,000 \\
\hline Outros & $22(28,6 \%)$ & $1(9,1 \%)$ & 0,275 \\
\hline \multicolumn{4}{|l|}{ Qualidade: } \\
\hline Queimação & $40(51,9 \%)$ & $6(54,5 \%)$ & 1,000 \\
\hline Aperto & $25(32,5 \%)$ & $4(36,4 \%)$ & 1,000 \\
\hline Outros Irradiação: & $16(20,8 \%)$ & $2(18,2 \%)$ & 1,000 \\
\hline Membro superior esquerdo & $48(62,3 \%)$ & $6(54,5 \%)$ & 0,743 \\
\hline Membro superior direito & $31(40,3 \%)$ & $4(36,4 \%)$ & 1,000 \\
\hline Ausente & $18(23,4 \%)$ & $3(27,3 \%)$ & 0,719 \\
\hline Pescoço & $13(16,9 \%)$ & $2(18,2 \%)$ & 1,000 \\
\hline Outros Sintomas associados: & $11(14,3 \%)$ & $3(27,3 \%)$ & 0,372 \\
\hline Sudorese & $50(64,9 \%)$ & $7(63,6 \%)$ & 1,0 \\
\hline Náuseas & $34(44,2 \%)$ & $4(36,4 \%)$ & 0,751 \\
\hline Vômitos & $20(26,0 \%)$ & $3(27,3 \%)$ & 1,000 \\
\hline Dispnéia & $20(26,0 \%)$ & $4(36,4 \%)$ & 0,483 \\
\hline Outros & $29(37,7 \%)$ & $3(27,3 \%)$ & 0,739 \\
\hline
\end{tabular}

Legenda:

+ Variáveis categóricas expressas como n (\%).

* Variáveis contínuas como média \pm desvio padrão.

$P^{\alpha}$ nível descritivo do teste exato de Fisher.

caracterizar-se como um fenômeno subjetivo, individual e influenciado por elementos sensitivos e emocionais ${ }^{(13-15)}$.

Nossos resultados vêm se contrapor a estudo realizado por Gondim e cols. ${ }^{(8)}$, que incluiu 80 pacientes com o objetivo de diferenciar a dor isquêmica do IAM entre pacientes diabéticos e não diabéticos. Os resultados mostraram que a presença de dor torácica na população de diabéticos foi de 20 (87\%) vs 57 (100\%) nos não diabéticos, com uma tendência dos pacientes diabéticos subestimarem mais a dor. Entretanto, estudo multicêntrico conduzido na Alemanha que incluiu 19.828 pacientes diabéticos e não diabéticos com sintomas de infarto, demonstrou que não houve diferença entre a frequência e a intensidade da dor torácica entre os dois grupos. Porém, os diabéticos tiveram uma maior ocorrência de dispnéia severa, estando relacionada a um estágio avançado de severidade da doença arterial coronariana, refletida por uma maior taxa de infartos prévios ${ }^{(16)}$.

Estes dados apresentam características semelhantes aos da amostra deste estudo, onde os pacientes diabéticos apresentaram uma maior prevalência de comorbidades associadas em relação aos não diabéticos, e embora com este quadro de cronicidade da doença e maior comprometimento do estado de saúde, não houve relação com a ausência e redução da intensidade da dor.

Essa associação do IAM e isquemia silenciosa foi avaliada também em estudo americano observacional realizado com 434.877 pacientes que pretendiam determinar a frequência com que os pacientes com IAM se apresentavam sem dor torácica, e mostrou que um a cada três pacientes com infarto não apresentaram dor torácica, sendo que os pacientes diabéticos representaram menos de um terço deste grupo. Concluindo que, embora o diabetes tenha sido um importante fator de risco para a apresentação atípica, outros fatores de risco estão associados com a ausência de dor, incluindo a idade avançada, sexo feminino, raça, história prévia de insuficiência cardíaca congestiva e acidente vascular encefálico ${ }^{(10)}$.

Estudo recente ${ }^{(17)}$ se propôs a investigar 290 pacientes com presença da angina pré-infarto como fator de proteção para eventos cardiovasculares após o primeiro infarto. Foram incluídos 143 pacientes idosos, com idade > 65 anos e 147 pacientes adultos com idade $<65$ anos. A angina pré-infarto manifestou-se tanto nos idosos como nos adultos $(44,4 \%$ vs $45,5 \%$, respectivamente), não havendo diferença entre os grupos. Ao avaliarem a presença da angina entre diabéticos e não diabéticos, também não foram encontradas diferenças significativas $(61,5$ vs $72,7 \%$ e $37,3 \%$ vs $38,3 \%$; $p=0,40)$. Estes dados também apresentam semelhanças a este estudo, onde não houve diferença significativa na presença e intensidade da dor entre pacientes diabéticos e não diabéticos.

Como limitação, destacamos a dificuldade de trabalhar com a dor, uma variável subjetiva e sujeita a diversas interpretações, dependendo da experiência e limiar individual de cada indivíduo. Observamos que, por vezes, a escala numérica apresentava-se como um obstáculo, devido à dificuldade de alguns pacientes em quantificar a dor, transformando-a em uma nota. Também podemos inferir como uma justificativa da reduzida amostra de diabéticos, o fato de que os pacientes com isquemia silenciosa, por não apresentarem componente 
anginoso, possam não ter procurado o serviço de emergência, e descoberto seu diagnóstico ao realizar exames laboratoriais ou de eletrocardiograma posterior ao evento.

Estudos futuros comparando nota da dor e significado/sentimento da dor torácica são de extrema relevância para observar se sentimento de morte/preocupação com familiares e/ou outros fatores possam interferir para aumento de limiar da dor. A percepção do profissional de saúde sob o significado da dor de cada paciente pode identificar esta situação e auxiliar em minimizar este sentimento, prevenindo assim, que estes influenciem ainda mais no quadro clínico do paciente ${ }^{(18)}$.

Apesar destas limitações, sabe-se que os pacientes portadores de DM devem ser atendidos com cautela pela equipe de saúde, principalmente no que tange a cardiopatias, uma vez que portadores de diabetes do tipo II têm uma alta prevalência de DAC. Um acompanhamento criterioso de pacientes com esta patologia é recomendado, mesmo sem a apresentação de sintomas típicos (angina, dispnéia e fadiga). Sugere-se frente a estas situações, que seja realizada uma estratificação do risco cardiovascular criteriosa, indicando quais exames mais apropriados para este tipo de paciente. Em casos de dor típica presente, é indicado que sejam realizados testes funcionais como cintilografia miocárdica e/ou ecocardiograma de estresse. Contudo, um aspecto que vem sendo debatido intensamente é a validade de realizar rastreamento para a presença de DAC em pacientes com DM sem sintomas de cardiopatia isquêmica, visto que o diabetes já é considerado um equivalente à presença de DAC prévia. Porém, a American Diabetes Association (ADA, 2008) não recomenda o rastreamento de
DAC apenas com base na presença de outros fatores de risco cardiovasculares e a American Heart Association (AHA, 2007) recomenda o rastreamento apenas ao iniciar um programa de exercícios vigorosos ${ }^{(19)}$.

Salienta-se a importância de uma rede de apoio possibilitando um processo educativo ao paciente com diabetes ampliando seus conhecimentos a fim de prevenir complicações cardiovasculares, possibilitando maior compreensão da doença e aquisição de hábitos saudáveis. A educação em diabetes deve estar centrada na equipe multidisciplinar, no sistema familiar, no paciente e nos equipamentos sociais ${ }^{(20)}$.

\section{CONCLUSÃO}

Os resultados deste estudo demonstram que a presença e intensidade da dor torácica entre pacientes diabéticos e não diabéticos acometidos de IAM são semelhantes. Destaca-se, entretanto, a importância do acompanhamento criterioso de pacientes diabéticos ao longo de sua vida, devido ao elevado risco de desenvolvimento de eventos cardiovasculares graves. Ressalta-se, também, que a equipe de saúde deve estar atenta a outros sintomas associados, que não somente os sintomas clássicos de angina, tanto em pacientes diabéticos como em não diabéticos. O desenvolvimento de novas pesquisas é fundamental para buscar mais evidências sobre os resultados obtidos, utilizando-se outras escalas de mensuração de dor, já existentes, com maior complexidade de caracteres, e com uma amostra maior de pacientes, possibilitando assim, uma melhor caracterização da dor no evento do IAM.

\section{REFERÊNCIAS}

1. Roglic G, Unwin N, Bennett P, Mathers C, Tuomilehto J, Nag S, et al. The burden of mortality attributable to diabetes: realistic estimates for the year 2000. Diabetes Care 2005;28:2130-5.

2. Meigs JB, Nathan DM, D'Agostino RB, Wilson PWF. Fasting and Postchallenge Glycemia and Cardiovascular Disease Risk The Framingham Offspring Study. Diabetes Care 2002;25: 1845-1850.

3. Andrade LJO, Cruz TRP, Daltro C, Soares FJ, França CS, Sampaio APQ, et al. Cintilografia de perfusão miocárdica em pacientes diabéticos tipo II com dor torácica atípica. Arq Bras Endocrinol Metab 2004;48(3):362-73.

4. Yusuf S, Hawken S, Ounpuu S, Dans T, Avezum A, Lanas $F$, et al. Effect of potentially modifiable risk factors associated with myocardial infarction in 52 countries (the INTERHEART study): case-control study. Lancet 2004;364(9438):937-52.

5. AFIRMAR Study Investigators. Risk factors for myocardial infarction in Brazil. Am Heart J 2003;146:331-8.

6. Amato R. Fisiopatologia e aspectos propedêuticos da dor torácica e cardíaca. In: Manual de Cardiologia SOCESP.
São Paulo:Atheneu; 2000. p22-5.

7. Braga JR, Santos IS, Flato UP, Guimarães HP, Avezum A. Impacto do Diabetes Mellitus na mortalidade em Síndromes Coronarianas Agudas. Arq Bras Endocrinol Metab 2007; 51(2):275-80.

8. Gondim LGP, Oliveira WA, Grossi SAA. A diferenciação da dor do infarto agudo do miocárdio entre pacientes diabéticos e não diabéticos. Rev Latinoam Enfermagem 2003;11(6):720-6.

9. Langer A, Freeman MR, Josse RG, Steiner G, Armstrong PW. Detection of silent myocardial ischemia in diabetes mellitus. Am J Cardiol 1991;67(13):1073-8.

10. Kentsch M, Rodemerk U, Gitt AK, Schiele R, Wienbergen $H$, Schubert J, et al. Angina intensity is not different in diabetic and non-diabetic patients with acute myocardial infarction. Z Kardiol 2003;92(10):817-24.

11. Sociedade Brasileira de Cardiologia. Diretrizes da Sociedade Brasileira de Cardiologia sobre Angina Instável e Infarto Agudo do Miocárdio sem Supradesnível do Segmento ST (II Edição, 2007). Arq Bras Cardiol 2007;89(4):e89-131. 
12. American Diabetes Association. Diagnosis and classification of diabetes mellitus. Diabetes Care 2008;31(Supl 1):S55-60.

13. Sousa FA, Hortense P. Mensuração da dor. In: Dor: $5^{\circ}$ sinal vital: reflexões e intervenções de enfermagem. Curitiba: Editora Maio; 2004.pg 75.

14. Pimenta CA, Teixeira MJ. Avaliação da dor. Medicina 1997;76(1):27-35.

15. Ministério da Saúde (Brasil). Direcção Geral de Saúde. Circular Normativa no 09/DGCG de 14 junho 2003. A dor como $5^{\circ}$ sinal vital. Registro sistemático da intensidade da dor.

16. Airaksinen KE. Silent coronary artery disease in diabetes - a feature of autonomic neuropath or accelerated atherosclerosis?. Diabetologia 2001;44(2):259-66.
17. Jiménez NMF, Muñoz GA, Ramirez MMA, Dominguez FA, Garcia AA, Gómez DJ, et al. Preinfarction angina prior to first myocardial infarction does not influence long-term prognosis: a retrospective study with subgroup analysis in elderly and diabetic patients. Clin Cardiol 2009;32(9):e62-5.

18. Araújo RD, Marques IR. Compreendendo o significado da dor torácica isquêmica de pacientes admitidos na sala de emergência. Rev Bras Enferm 2007;60(6):676-80.

19. Triches C, Schaan B, Gross JL, Azevedo MJ. Complicações macrovasculares do diabetes melito: peculiaridades clínicas, de diagnóstico e manejo. Arq Bras Endocrinol Metabol 2009;53(6):698-708.

20. Zanetti ML, Biagg MV, Santos MA, Péres DS, Teixeira CRS. O cuidado à pessoa diabética e as repercussões na família. Rev Bras Enferm 2008;61(2):186-92. 